\title{
Forequarter amputation in a patient with locally advanced recurrent breast carcinoma
}

\author{
René Aloisio da Costa Vieira1,2,3 (D), Eduardo Areas Toller ${ }^{4}$ (D), \\ Andréa Moreno Morgan ${ }^{1,5}$ (i), Idam de Oliveira-Junior ${ }^{2,5}$ (i)
}

\section{ABSTRACT}

Forequarter amputation (FQA) involves the removal of the upper limb, clavicle, and scapula and is indicated for the resection of primary or metastatic tumors invading the axillary neurovascular bundle. Reports on breast cancer have associated FQA with the primary resection of a locally advanced tumor, resection of recurrent disease, brachial plexus injury, Stewart-Treves syndrome, or sarcoma secondary to breast cancer irradiation. We described a case of recurrent breast carcinoma with curative-intent surgery. The surgery aimed at locoregional control and improvement in the quality of life. The literature is scarce on the topic, discussing the multiple aspects related to the indication of FQA for breast cancer patients. This report presents the first case described in Latin American literature.

KEYWORDS: Disarticulation; Amputation; Breast neoplasms.

\section{INTRODUCTION}

Surgeries that treat tumors of the shoulder girdle are extensive. Forequarter amputation (FQA) involves the removal of the upper limb, clavicle, and scapula and is indicated for the resection of primary or metastatic tumors invading the axillary neurovascular bundle. Although often described in cases of Stewart-Treves syndrome, post-mastectomy sarcomas, and lymphedema, this surgery is rarely reported in carcinomas. Reports on breast cancer have associated FQA with the primary resection of a locally advanced tumor ${ }^{1}$, resection of recurrent disease $e^{2-5}$, brachial plexus injury ${ }^{5}$, Stewart-Treves syndrome ${ }^{6}$, or sarcoma secondary to breast cancer irradiation ${ }^{7.8}$. The literature is scarce on the topic, and the surgery aimed at locoregional control and improvement in the quality of life, justifying this publication.

\section{CASE REPORT}

Female, 73years old, clinical stage T4bN3M0, associated with extensive and limiting lymphedema of the right upper limb (Figure 1A).
Although hypertension was her only comorbidity, the patient was clinically classified as grade 2 in the Eastern Cooperative Oncology Group (ECOG) Performance Status. The biopsy revealed a triple-negative invasive ductal carcinoma of histological grade 3. Initially, the patient underwent two cycles of neoadjuvant chemotherapy with paclitaxel, not responding to therapy and developing febrile neutropenia. Chemotherapy was suspended due to the worsening of her general condition (ECOG grade 3), asthenia, and inappetence. In this context, the treatment chosen was surgery, and the patient was submitted to a right-sided Halsted mastectomy, considered R1 (minimal microscopic disease) because of the disease located along the brachial plexus (Figure 1). Adjuvant radiotherapy was considered for local control, but the presence of surgical wound dehiscence prevented this treatment. Two months later, she showed visible macroscopic recurrence next to the skin of the axillary fossa, leading to the performance of an $\mathrm{R} 1$ resection of the region affected by the neoplasm, adjacent to the dehiscence area, with external oblique myocutaneous rotation flap to close the surgical wound

\footnotetext{
${ }^{1}$ Graduate Program in Oncology, Hospital do Câncer de Barretos - Barretos (SP), Brazil.

${ }^{2}$ Graduate Program in Gynecology, Obstetrics, and Mastology, School of Medicine of Botucatu - Botucatu (SP), Brazil.

${ }^{3}$ Department of Surgery, Mastology Division, Hospital do Câncer de Muriaé, Fundação Cristiano Varella - Muriaé (MG), Brazil.

${ }^{4}$ Department of Orthopedics, Hospital do Câncer de Barretos - Barretos (SP), Brazil.

${ }^{5}$ Department of Mastology and Breast Reconstruction, Hospital do Câncer de Barretos - Barretos (SP), Brazil.

*Corresponding author: posgrad@hcancerbarretos.com.br

Conflict of interests: nothing to declare.

Received on: 07/29/2019. Accepted on: 12/25/2019
} 
and provide conditions for adjuvant radiotherapy. She presented new local dehiscence and, in the healing stage, new macroscopic local recurrence (Figures 1 and 2).

Thus, due to the impossibility of administering adjuvant radiotherapy and the early recurrence, FQA was chosen for local control and potential improvement in her quality of life, since the upper limb was no longer functional. FQA was considered R0 (complete resection; Figure 2), and the surgical progress was satisfactory, allowing the start of adjuvant radiotherapy. The patient was questioned about her general quality of life (scores from 1-terrible to 7-great) in the preoperative period, as well as one and three months after surgery. She reported a score of 3 in the preoperative period and 5 in the first and third months. Four months after surgery, she was asymptomatic but showed weight loss of $18 \mathrm{~kg}$, and developed local recurrence metastasis and lung metastasis, being referred to exclusively palliative treatment (Figure 3). Seven months after the FQA, the patient died of pulmonary metastatic disease. FQA has improved her quality of life.
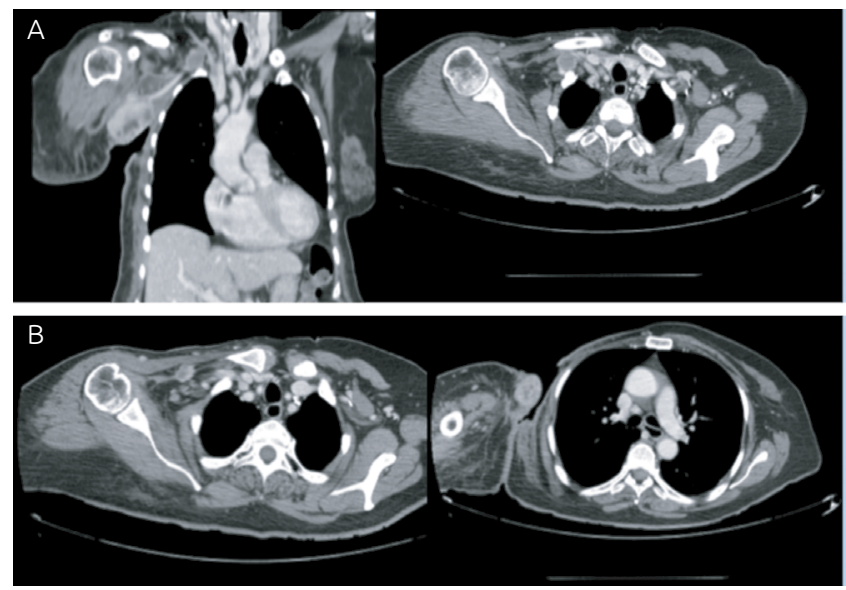

Figure 1. Chest computed tomography (A) pre-treatment;

(B) after breast lesion resection with minimal residual extrathoracic disease.
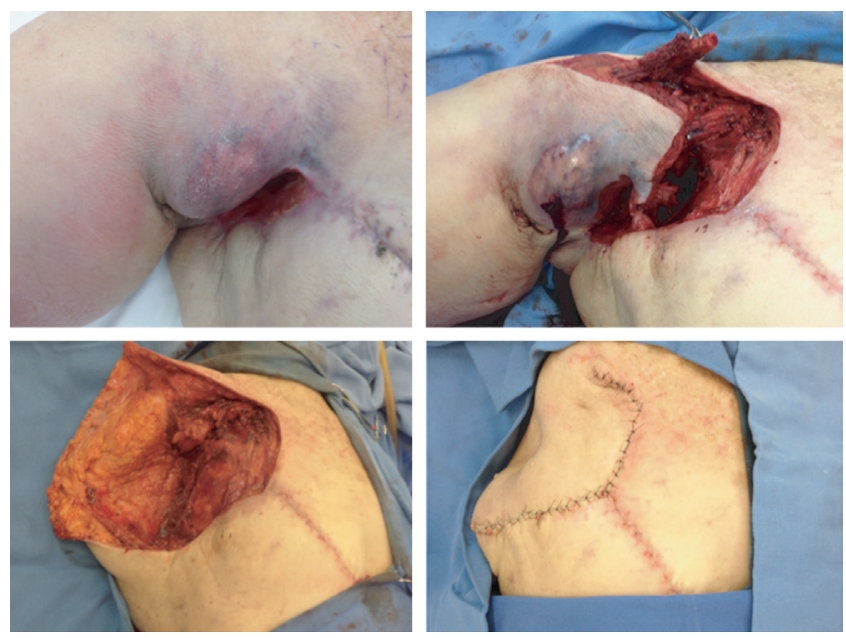

Figure 2. Forequarter amputation.

\section{DISCUSSION}

In patients submitted to axillary treatment, recurrence is a rare phenomenon, and, even with surgical treatment, the $\mathrm{R} 1$ resection ${ }^{9}$ is not often complete. These patients require adjuvant therapies, such as chemotherapy and radiotherapy ${ }^{9,10}$, for long-term control of the disease. In some individuals, FQA may be necessary for locoregional control ${ }^{2,4}$.

FQA is often performed in cases of tumor of the shoulder girdle ${ }^{11}$. This procedure is usually carried out with curative or palliative intent, allowing locoregional control of the disease and improving the quality of life. Reports on breast cancer have associated FQA with the primary resection of a locally advanced tumor $^{1}$, resection of recurrent disease ${ }^{2-5}$, brachial plexus injury ${ }^{5}$, Stewart-Treves syndrome ${ }^{6}$, or sarcoma secondary to breast cancer irradiation ${ }^{7,8}$. In series of this type of surgery, the association with breast cancer represents, on average, $12.5 \%$ of the causes ${ }^{11}$, an incidence that increases $(37.5 \%)$ when considering the presence of metastatic disease ${ }^{12}$. Recurrence is its main indication ${ }^{2-5,12}$ with palliative intent ${ }^{3,5}$. The literature is scarce on the topic, and we found no cases described in Latin American literature.

Despite the radical nature of the surgery, it allows locoregional control, improvement in symptoms and quality of life, and prolongation of the disease-free interval, which justify its performance in selected cases with curative or palliative intent $t^{2,3,5}$. Similarly, this procedure should be considered for patients with brachial plexus injury, neurovascular involvement, and upper limb dysfunction ${ }^{5}$.

In the present case, the initial surgery showed the presence of disease along the brachial plexus, and, at first, surgery was not indicated, as radiotherapy was contemplated for local control. Unfortunately, the patient progressed to local dehiscence. Initially, the abdominal oblique flap was considered for primary closure. The new dehiscence, the impossibility of administering other adjuvant therapy, and the local progression of the disease led to the performance of a curative-intent FQA, but the patient
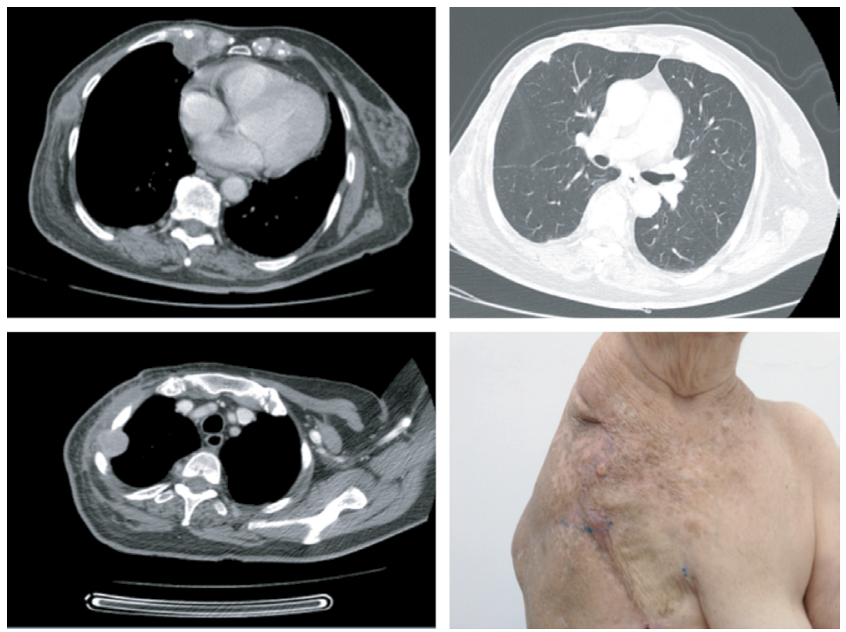

Figure 3. Local and lung recurrence. 
died seven months later due to the progression of the lung disease. Usually, FQA is indicated for patients with distant recurrence and prolonged disease-free interval ${ }^{3}$; however, the complications and the clinical condition of the patient led to surgical treatment being the only option for local control.

One of the main points to consider with respect to FQA is the closure of the resected area, which can be done with skin grafts, reuse of part of the skin of the limb, and myocutaneous rotation flaps ${ }^{2,3,5}$. The complication rate is relatively low and usually associated with skin necrosis, local dehiscence, and pleural effusion $^{2-5}$. In this case, the local flaps used originated from the healthy skin of the shoulder, careful of the small area of local dehiscence, controlled with resuture and dressings.

FQA has not been evaluated yet regarding the breast cancer tumor subtype. Triple-negative tumors show worse behavior, but studies involving FQA did not assess this fact. Survival is better in curative-intent treatments, with a mean of 23 months, decreasing to 13 months in palliative ones ${ }^{3}$, which fully justifies the surgery in selected cases. In this patient with a triple-negative tumor, FQA was considered curative because of the R0 resection; however, her clinical conditions were poor. The lack of adjuvant therapy and the aggressive nature of the tumor influenced the local recurrence and the short disease-free interval, resulting in limited survival.

\section{CONCLUSION}

FQA is an exceptional procedure for patients with recurrent breast carcinoma. It is associated with low surgical morbidity and mortality and should be considered, even if with palliative intent, for prolonging the disease-free interval and improving symptoms of specific diseases and the quality of life.

\section{AUTHORS' CONTRIBUTION}

R.A.C.V.: study concept, data curation, formal analysis, methodology, project management.

E.A.T.: data curation, research, methodology.

A.M.M.: methodology.

I.O.-Jr.: formal analysis, methodology.

All authors contributed to the writing of the original manuscript, in addition to reviewing and editing the article.

\section{REFERENCES}

1. Ayvaz M, Yilgor C, Mermerkaya UM, Konan A, Sonmez E, Acaroglu RE. Simultaneous forequarter amputation and radical mastectomy for metastatic breast carcinoma in a male patient: a case report. J Korean Surg Soc. 2011;81(Supl. 1):S6S11. https://dx.doi.org/10.4174\%2Fjkss.2011.81.Suppl1.S6

2. Goodman MD, McIntyre B, Shaughnessy EA, Lowy AM, Ahmad SA. Forequarter amputation for recurrent breast cancer: a case report and review of the literature. J Surg Oncol. 2005;92(2):134-41. https://doi.org/10.1002/jso.20337

3. Pundi KN, AlJamal YN, Ruparel RK, Farley DR. Forequarter amputation for recurrent breast cancer. Int J Surg Case Rep. 2015;11:24-8. https://dx.doi.org/10.1016\%2Fj.ijscr.2015.04.018

4. Tsai CH, Tzeng HE, Juang WK, Chu PG, Fann P, Fong YC, et al. Curative use of forequarter amputation for recurrent breast cancer over an axillary area: a case report and literature review. World J Surg Oncol. 2014;12:346. https://doi.org/10.1186/1477-7819-12-346

5. Behnke NK, Crosby SN, Stutz CM, Holt GE. Periscapular amputation as treatment for brachial plexopathy secondary to recurrent breast carcinoma: a case series and review of the literature. Eur J Surg Oncol. 2013;39(12):1325-31. https://doi. org/10.1016/j.ejso.2013.10.005

6. Roy P,Clark MA, Thomas JM. Stewart-Treves syndrome--treatment and outcome in six patients from a single centre. Eur J Surg Oncol. 2004;30(9):982-6. https://doi.org/10.1016/j.ejso.2004.07.027
7. Borman H, Safak T, Ertoy D. Fibrosarcoma following radiotherapy for breast carcinoma: a case report and review of the literature. Ann Plast Surg. 1998;41(2):201-4. https://doi. org/10.1097/00000637-199808000-00015

8. Doherty MA, Rodger A, Langlands AO. Sarcoma of bone following therapeutic irradiation for breast carcinoma. Int J Radiat Oncol Biol Phys. 1986;12(1):103-6. https://doi. org/10.1016/0360-3016(86)90422-0

9. de Boer R, Hillen HF, Roumen RM, Rutten HJ, van der Sangen MJ, Voogd AC. Detection, treatment and outcome of axillary recurrence after axillary clearance for invasive breast cancer. Br J Surg. 2001;88(1):118-22. https://doi.org/10.1046/j.1365-2168.2001.01637.x

10. Newman LA,HuntKK, BuchholzT, Kuerer HM, Vlastos G, Mirza $\mathrm{N}$, et al. Presentation, management and outcome of axillary recurrence from breast cancer. Am J Surg. 2000;180(4):252-6. https://doi.org/10.1016/s0002-9610(00)00456-6

11. Rickelt J, Hoekstra H, van Coevorden F, de Vreeze R, Verhoef $\mathrm{C}$, van Geel AN. Forequarter amputation for malignancy. Br J Surg. 2009;96(7):792-8. https://doi.org/10.1002/bjs.6555

12. Wittig JC, Bickels J, Kollender Y, Kellar-Graney KL, Meller I, Malawer MM. Palliative forequarter amputation for metastatic carcinoma to the shoulder girdle region: indications, preoperative evaluation, surgical technique, and results. J Surg Oncol. 2001;77(2):105-13; discussion 114. https://doi.org/10.1002/jso.1079 